\title{
Burden of Uterine Rupture and Its Determinant Factors in Ethiopia: A Systematic Review and Meta-analysis
}

\author{
Dagne Addisu $\mathbb{D},{ }^{1}$ Maru Mekie $\mathbb{D}^{1},{ }^{1}$ Abenezer Melkie $\mathbb{D},{ }^{1}$ and Worku Necho $\mathbb{}^{2}$ \\ ${ }^{1}$ Department of Midwifery, College of Health Sciences, Debre Tabor University, Debre Tabor, Ethiopia \\ ${ }^{2}$ Department of Maternity Health Nursing, College of Health Sciences, Debre Tabor University, Debre Tabor, Ethiopia
}

Correspondence should be addressed to Dagne Addisu; addisudagne7@gmail.com

Received 29 July 2020; Revised 28 October 2020; Accepted 5 November 2020; Published 16 November 2020

Academic Editor: Carla B Andreucci

Copyright (C) 2020 Dagne Addisu et al. This is an open access article distributed under the Creative Commons Attribution License, which permits unrestricted use, distribution, and reproduction in any medium, provided the original work is properly cited.

\begin{abstract}
Background. Uterine rupture is a life-threatening obstetrical emergency, which results in serious undesired maternal and perinatal complications in resource-limited countries, mainly in Ethiopia. The prevalence, determinants, and outcomes of uterine rupture described by different studies were highly inconsistent in Ethiopia. Therefore, this systematic review and meta-analysis is aimed at estimating the pooled prevalence, determinants, and adverse outcomes of uterine rupture in Ethiopia. Methods. Studies were searched from international databases (PubMed/MEDLINE, HINARI, Cochrane library, Google Scholar, and web of science) and Ethiopian universities' digital libraries (Addis Ababa University, University of Gondar, and Harameya University). All observational studies were included. A total of 15 studies that fulfill the inclusion criteria were included in this meta-analysis. Data were extracted by two reviewers and exported to STATA version 11 for analysis. The $I^{2}$ statistics were used to assess heterogeneity across the studies. Publication bias was examined by using Egger's test and funnel plot. The pooled prevalence of uterine rupture and its outcomes were estimated by using a random effects model. The associations between determinants and uterine rupture were evaluated by using both random and fixed-effect models. Results. In this meta-analysis, a total of 15 studies with 92,394 study participants were involved. The pooled prevalence of uterine rupture was 2.37\% in Ethiopia. Obstructed labor $(\mathrm{OR}=3.03 ; 95 \% \mathrm{CI}=2.52,3.63)$, lack of antenatal care follow-up $(\mathrm{OR}=5.79 ; 95 \% \mathrm{CI}=2.47,13.61)$, duration of labor $>24$ hours $(\mathrm{OR}=3.75 ; 95 \% \mathrm{CI}=2.24,6.29)$, grand multipara $(\mathrm{OR}=10.79 ; 95 \% \mathrm{CI}=4.77,24.40)$, and being rural residency $(\mathrm{OR}=7.17 ; 95 \%$ $\mathrm{CI}=3.90,13.16)$ were significantly associated with uterine rupture. Conclusion. The overall prevalence of uterine rupture was high in Ethiopia. Obstructed labor, lack of antenatal care follow-up, duration of labor $>24$ hours, grand multipara, and rural residency were determinants of uterine rupture. This study implies the need to develop plans and policies to improve antenatal care follow-up and labor and delivery management at each level of the health system.
\end{abstract}

\section{Introduction}

Uterine rupture is a serious obstetric emergency, whereby the uterine wall is disrupted and loss its integrity either to some extent or totally during pregnancy, delivery, or immediately after birth $[1,2]$. The disruption extends to the uterine serosa and occasionally involving the bladder or broad ligament in the case of complete uterine rupture [3].

Various direct and indirect factors have increased the risk of uterine rupture. In high-income countries, uterine rupture most commonly occurred in scarred uterus [4], while in resource-limited countries, uterine rupture mainly occurred in unscarred uterus. Obstructed labor, grand multiparty, inadvisable obstetric procedures, lack of ANC, and advanced age were the major determinants of uterine rupture in resource-limited countries. Beside to this, misuse of uterotonic drugs and delays (delay in reaching health facilities, delay in deciding for referral, and delay in seeking appropriate care) also leads to uterine rupture [5-10].

The incidence of uterine rupture varies in different parts of the world. It is a rare obstetric event in high-income countries and complicates around $0.03 \%$ pregnancies [4]. A large population-based studies in European countries showed that the incidence of uterine rupture was $0.036 \%$ in Belgium [4], $0.091 \%$ in Sweden [11], 0.019\% in United Kingdom [10], and $0.059 \%$ in Netherland [12]. In low- and middle-income 
countries, the prevalence of uterine rupture for women with previous caesarean section was $1 \%$, whereas for women without previous caesarean section, the overall rate was below 1 per 1000 [13-15].

Globally, nearly 295,000 women died annually as a result of complications of pregnancy and childbirth. The majority of these happen in resource-limited countries mainly in sub-Sahara Africa, which covers more than $94 \%$ of the burden [16]. Uterine rupture accounts $8 \%$ of this maternal globally and $90 \%$ maternal deaths in sub-Sahara Africa countries [5, 17-19]. In Ethiopia, uterine rupture is the leading cause of obstetric hemorrhage and accounts up to $36 \%$ of maternal deaths $[20,21]$.

Uterine rupture also leads to grave maternal and fetal complications both in high income and resource-limited countries including Ethiopia [19, 22]. It leads to severe perinatal asphyxia, perinatal death, massive maternal bleeding necessitating massive transfusion, bladder injury, foot drop, psychological disorder, obstetric fistula, anemia, infertility, and hysterectomy [4, 23-25]. Globally, the perinatal mortality rate as a result of uterine rupture was nearly $11.2 \%$ [25].

Ethiopia has implemented different strategies to decrease maternal mortality and morbidity. Some of those approaches were increasing accessibility of emergency obstetrics care, improve ANC follow-up, established maternal waiting homes, improve institutional delivery, and use of partograph during labor. However, uterine rupture is still a common obstetric problem in the country $[26,27]$.

Even if there are many fragmented primary studies on the prevalence, predictors, and outcomes of Uterine rupture in Ethiopia, the overall prevalence and adverse outcomes are not yet known at the national level [1, 9, 17, 28-34]. Besides, the majority of the literature showed a difference in the magnitude of uterine rupture over time and across geographical areas that ranges from $0.9 \%$ [30] to $16.68 \%$ [28]. Likewise, a discrepancy was observed among those studies while reporting the major determinants of uterine rupture. Hence, this systematic review and meta-analysis intended to estimate the overall national prevalence, determinants, and adverse outcomes of uterine rupture in Ethiopia. The result of this study may help policy or decision-makers and clinicians at different levels of designing appropriate prevention and control strategies for uterine rupture.

\section{Materials and Methods}

2.1. Study Design. A systematic review and meta-analysis was done to estimate the pooled prevalence, determinants, and adverse outcomes of uterine rupture in Ethiopia. The result of this meta-analysis was reported via systematic review and meta-analyses reporting PRISMA guidelines. Protocol registration was no done in this systematic review and meta-analysis.

2.2. Databases and Search Strategies. Studies were searched exhaustively by three authors (DA, MM, and WN) from international databases (PubMed/MEDLINE, HINARI, Cochrane library, Google Scholar, and web of science) by using comprehensive searching strategies. Initially, pretested search strategies were developed to retrieve all pertinent studies. First, articles were searched by examining the full titles ("incidence and determinants of uterine rupture in Ethiopia") and then keywords ("prevalence," "incidence," "magnitude," "determinants," "determinant factors," "associated factors," "uterine rupture," "ruptured uterus," "uterine," "rupture," "uterine separation," and "Ethiopia"). These keywords were used independently and in combination using Boolean operators "OR" or "AND." Besides to this, studies were also searched from the reference lists of all included studies to find any other missed studies by our searching strategies. Furthermore, to find pertinent unpublished studies, Ethiopian universities' digital libraries were searched (Addis Ababa University, University of Gondar, and Harameya University). Search details for PubMed were ("epidemiology"[Subheading] OR “epidemiology”[All Fields] OR "incidence" [All fields] OR "incidence" [MeSH Terms] OR "magnitude"[All fields] or "magnitude" [MeSH Terms] OR "prevalence"[All Fields] OR "prevalence"[MeSH Terms]) AND ("associated factors"[All Fields] OR "associated factors"[MeSH Terms]) OR “determinant factors"[All fields] OR "determinant factors"[MeSH Terms] AND ("uterine rupture"[MeSH Terms] OR ("uterine"[All Fields] AND "rupture"[All Fields]) OR "uterine rupture"[All Fields]) AND ("Ethiopia”[MeSH Terms] OR “Ethiopia”[All Fields]). The searching periods were from January 1, 2000 to June 8, 2020 G.C were included in this review (Table 1).

2.3. Eligibility Criteria. This systematic review and metaanalysis included studies that meet the following criteria: (1) studies done in Ethiopia and reporting the prevalence or incidence and/or outcomes and/or determinants of uterine rupture among deliveries; (2) studies published between January 1, 2000 and June 8, 2020 G.C; (3) unpublished studies done between January 1, 2000 and June 8, 2020 G.C; (4) all observational study designs (i.e., cross-sectional, cohort, and case-control study designs) that report the prevalence, outcomes, and associated factors of uterine rupture. Studies which were not fully accessible from the database or failed to get a response from the corresponding author within 2 weeks of contact by email were excluded. Inabilities of assessing the quality of studies without full content were the reason for exclusion.

2.4. Measurement of Outcome Variables. This systematic review and meta-analysis had three outcomes. The primary outcome was uterine rupture, which was defined as disruption and loss of uterine wall integrity either partially or completely during delivery or immediately after delivery [1]. Adverse outcomes and determinants of uterine rupture were the secondary and tertiary outcomes, respectively.

2.5. Study Selection and Quality Assessment. All retrieved articles were exported to Endnote 7 reference manager. Then, duplicated studies were removed. The remaining studies were screened for its relevancy, outcome of interest, and availability of full text by three reviewers (DA, MM, and AM). Studies with irrelevant titles and without full text were removed. Finally, studies that fulfilled the inclusion criteria 
TABle 1: Shows examples of searching strategies for PubMed and Google Scholar databases to assess the prevalence, determinants, and adverse outcomes of uterine in Ethiopia.

\begin{tabular}{|c|c|c|}
\hline Databases & Searching terms & $\begin{array}{l}\text { Number } \\
\text { of } \\
\text { studies }\end{array}$ \\
\hline PubMed/MEDLINE & 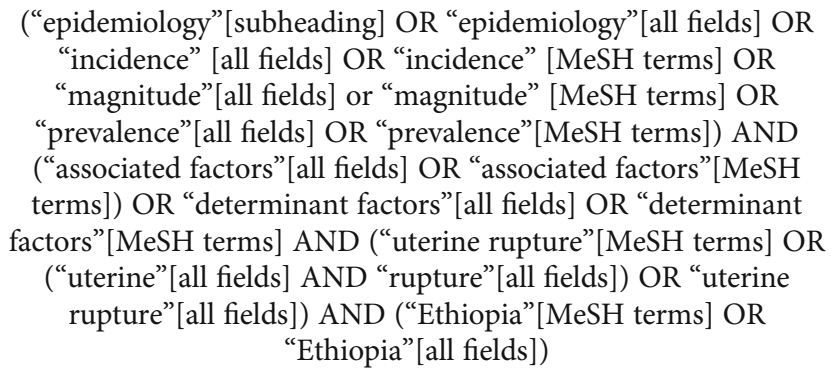 & 114 \\
\hline Google scholar & $\begin{array}{l}\text { "Prevalence" or "magnitude" or "incidence" and "associated } \\
\text { factors" or "determinant factors" and "uterine rupture" or } \\
\text { "ruptured uterus" and "Ethiopia" }\end{array}$ & 4 \\
\hline Other databases & & 189 \\
\hline $\begin{array}{l}\text { Gray literature (specifically unpublished theses report, } \\
\text { doctoral theses, research reports, deposited papers, and call } \\
\text { for papers) }\end{array}$ & & 6 \\
\hline Total searched articles & & 313 \\
\hline Finally, fulfill the eligibility criteria for our review & & 15 \\
\hline
\end{tabular}

were included. The quality of the studies was assessed by two reviewers (DA and $\mathrm{MM}$ ) individually using NewcastleOttawa quality assessment Scale (NOS) [35]. Disagreements during quality assessment were resolved by the third reviewer (AM). During disagreement, the third reviewer evaluated the articles and resolved inconsistencies through discussion and consensus. The following items were used to appraise crosssectional studies: (1) representativeness of the sample; (2) nonrespondents; (3) ascertainment of the exposure (risk factor); (4) the subjects in different outcome groups are comparable, based on the study design or analysis; confounding factors are controlled; (5) assessment of the outcome; (6) statistical test.

The following items were used for quality assessment of cohort study: (1) representativeness of the exposed cohort; (2) selection of the nonexposed cohort; (3) ascertainment of exposure; (4) demonstration that outcome of interest was not present at the start of the study; (5) comparability of cohorts based on the design or analysis; (6) assessment of outcome; (7) was follow up long enough for outcomes to occur; (8) adequacy of follow-up of cohorts: complete follow-up or subjects lost to follow up unlikely. The following items were used for appraising case-control studies: (1) adequate case definition; (2) representativeness of the cases; (3) selection of controls; (4) definition of controls; (5) comparability of cases and controls based on the design or analysis; (6) ascertainment of exposure; (7) same method of ascertainment for cases and controls; (8) nonresponse rate.

Articles that scored $\geq 7$ points out of 9 for cross-sectional, $\geq 9$ points out of 10 for case-control, and $\geq 9$ points out of 13 for cohort studies were considered to be high quality and included in this systematic review and meta-analysis.
2.6. Data Extraction. All necessary data were extracted from 15 primary studies by two reviewers (DA and MM) individually using predesigned data abstraction form. This form included the last name of the first author, year of publication, study area, study design, study region, study period, sample size, sampling technique, prevalence of uterine rupture with 95\% CI, adjusted odds ratio (AOR) with 95\% CI for identified risk factors, and adverse outcomes with its magnitude (i.e., anemia, blood transfusion, vesicovaginal fistula, wound infection, maternal death, relaparotomy, puerperal sepsis, and stillbirth). Disagreements during the data extraction were resolved by the third reviewer (AM). In the case of disagreement, the third reviewer evaluates the articles and resolved inconsistencies through discussion and consensus.

2.7. Data Processing and Analysis. All necessary data were extracted and analyzed by using Microsoft Excel and STATA version 11, respectively. The presence of heterogeneity across the studies was evaluated by using $I^{2}$ statistics [36]. The result of the $I$-squared test indicated the presence of marked heterogeneity between the studies $\left(I^{2}=97.4 \%\right.$ and $\left.p<0.001\right)$. A random effects model was applied to determine the overall prevalence of uterine rupture $[37,38]$. Subgroup analysis was done to minimize the random variations between the point estimates of primary studies by using different study characteristics (i.e., based on study region where the study was done, year of publication, and sample size). However, heterogeneity is still observed across the studies. Publication bias was evaluated subjectively by using a funnel plot and objectively by using Egger's regression test [39]. The result of funnel plot and Egger's regression test showed the presence of publication bias across the studies $(p$ value $=0.003)$. The 


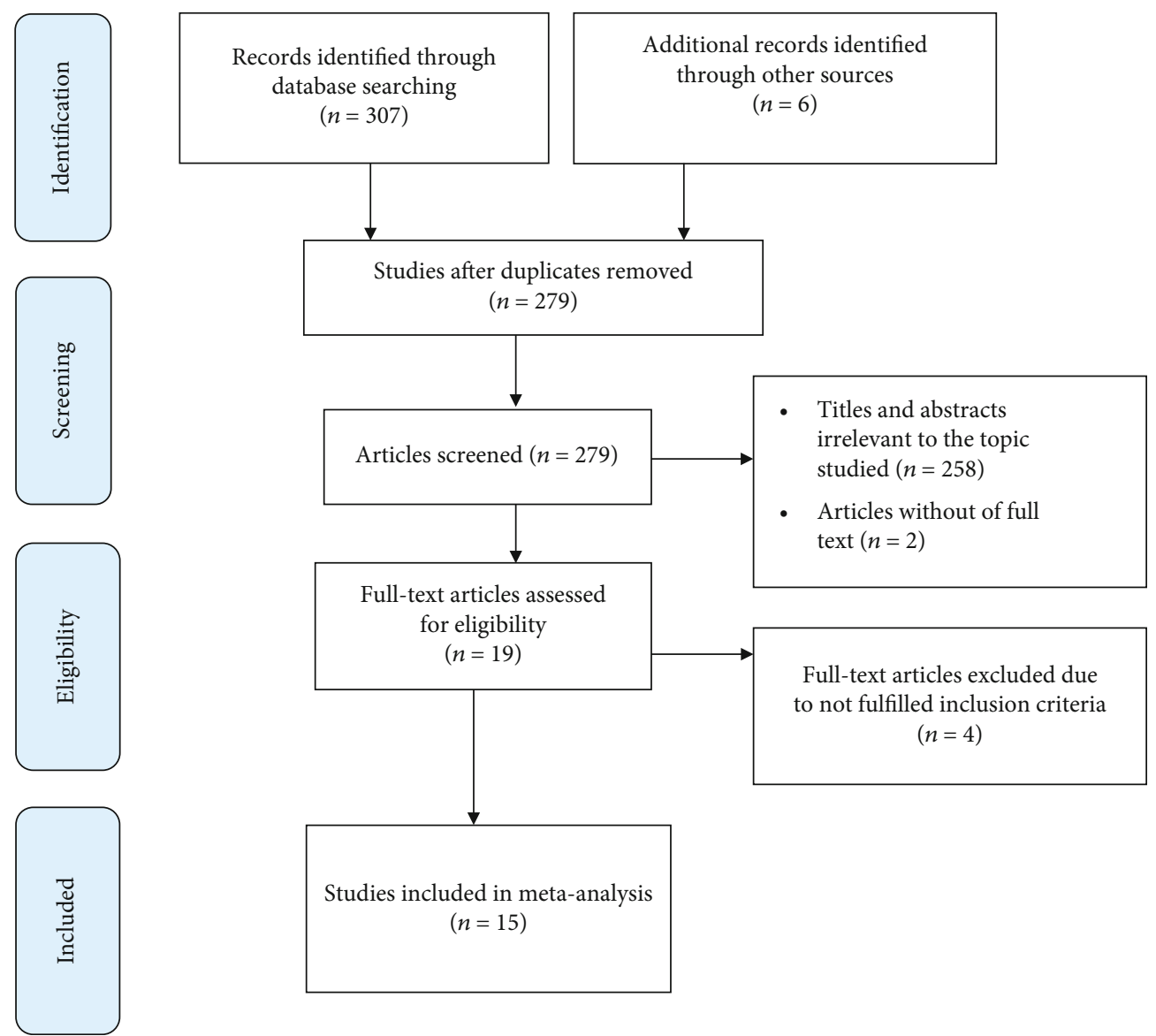

Figure 1: Flow chart describing study selection for systematic review and meta-analysis of the prevalence, determinants, and adverse outcomes of uterine rupture in Ethiopia.

Duval and Tweedie nonparametric trim and fill analysis was done to correct publication bias among the studies. Adverse outcomes and determinants of uterine rupture were examined by using both random and fixed-effect models.

\section{Result}

In this systematic review and meta-analysis, a total of 313 articles were retrieved from the international databases and Ethiopian universities' digital libraries. All identified studies were exported to Endnote 7 reference manager. Then, thirty-four duplicated studies were removed. Two hundred seventy-nine studies were screened for its relevancy, outcome of interest, and availability of full text. Two hundred fiftyeight articles were removed due to irrelevant titles, and two studies were excluded due to the inaccessibility of full text. Nineteen full-text articles were assessed for eligibility. Four studies did not fulfill the inclusion criteria (mainly study period and year of publication). Finally, fifteen studies that fulfilled the inclusion criteria were included (Figure 1).

\section{Characteristics of Included Studies}

In this systematic review and meta-analysis, a total of 15 primary studies that report the prevalence/incidence/magnitude, determinants/associated factors, and adverse outcomes of uterine rupture were included to estimate the national prevalence, determinants, and adverse outcomes of uterine rupture in Ethiopia. All studies were published in different international journals between 2004 and 2020 G.C. Regarding study design, nine studies were cross-sectional [1, 17, $23,28-30,32-34]$, five studies were case-control studies [5, $9,18,40,41]$, and one study was cohort study [31]. In this meta-analysis, a total of 92,394 study participants were involved. In the present meta-analysis, four regions in Ethiopia were represented. Seven studies were from Amhara region $[1,23,28,29,32,33,41]$, three from Oromiya region $[18,34,40]$, three from Southern Nations, Nationalities, and Peoples Region (SNNPR) [9, 17, 31], and two from Tigray region $[5,30]$ (Table 2 ).

\section{The Pooled Prevalence of Uterine Rupture in Ethiopia}

Eleven primary studies were used to estimate the pooled prevalence of uterine rupture in Ethiopia. Accordingly, the pooled prevalence of uterine rupture was $2.37 \%$ (1.83 and 2.91). High heterogeneity was observed across studies $\left(I^{2}=97.4 \%\right.$ and $\left.p \leq 0.001\right)$. Therefore, the overall pooled prevalence of uterine rupture was determined by using random effects model. The highest prevalence of uterine rupture 
TABLE 2: Shows descriptive summary of 15 studies reporting the prevalence, determinants, and adverse outcomes of uterine rupture in Ethiopia.

\begin{tabular}{lccccc}
\hline Author & Publication year & Region & Study area & Study design & Sample size \\
& & & Number of \\
uterine rupture
\end{tabular}

was reported at referral hospitals in Amhara region done by Getahun et al. (16.67\%) [28] followed by Taddel et al. (8.73\%) (32) while the lowest prevalence was reported by Berhe et al. [30] and Ahmed et al. [1] in Mekelle and Felege Hiwot referral hospitals, respectively (0.91\%) (Figure 2).

\section{Subgroup Analysis}

In this systematic review and meta-analysis, marked heterogeneity was observed across the studies $\left(I^{2}=97.4 \%\right.$ and $p \leq$ $0.001)$. Therefore, subgroup analysis was done based on publication year, study design, sample size, and the region where the study was conducted. However, heterogeneity is still observed across the studies. Based on region, the highest prevalence of uterine rupture was seen in Amhara (5.38\%) followed by Southern Nations, Nationalities, and Peoples Region (SNNPR) (1.88\%), Oromiya region (1.20), and then Tigray region $(0.91 \%)$. Regarding to publication year, the highest prevalence was observed in those studies published after 2017 G.C (2.83\%). According to sample size, the highest prevalence was observed in those studies which had sample size $\leq 6000(4.53 \%)$ (Table 3$)$.

\section{Publication Bias}

In this meta-analysis, publication biases among the included studies were examined by using both funnel plots and Egger's regression test. The results of funnel plots showed an asymmetric shape, which indicates the presence of publication bias among those included studies (Figure 3). Objective assessments of publication bias by Egger's regression test also showed the presence of publication bias across studies ( $p$ value $=0.003)$. Hence, the Duval and Tweedie nonparametric trim and fill analysis was done to correct publication bias among the studies. Accordingly, publication bias was cor- rected when five missed studies were filled in the funnel plot by trim and fill analysis (Figure 4).

\section{Adverse Outcomes of Uterine Rupture}

Uterine rupture leads to undesirable adverse maternal and fetal outcomes in Ethiopia. In this systematic review and meta-analysis, adverse maternal and perinatal outcomes were examined by using nine studies that report outcomes of uterine rupture. Accordingly, anemia, blood transfusion, vesicovaginal fistula, wound infection, maternal death, relaparotomy, puerperal sepsis, and stillbirth were the major adverse outcomes of uterine rupture in Ethiopia. Stillbirth was the leading complications of uterine rupture with a magnitude of $95.01 \%$ (92.43 and 97.60) followed by anemia with a magnitude of $64.34 \%$ (33.06 and 95.61). Both random and fixed-effect models were used to assess the prevalence of each outcome (Table 4).

\section{Determinants of Uterine Rupture in Ethiopia}

9.1. The Association between Obstructed Labor and Uterine Rupture. The effect of obstructed labor on uterine rupture was evaluated by using four studies that report the association of two variables $[5,28,32,41]$. The results of this meta-analysis revealed that obstructed labor was significantly associated with uterine rupture. The likelihood of uterine rupture was 3.03 times higher in those laboring mothers who had obstructed labor as compared to those who did not have obstructed labor $(\mathrm{OR}=3.03 ; 95 \% \mathrm{CI}=2.52,3.63)$. There was no heterogeneity between the studies $\left(I^{2}=0.0 \%\right.$ and $p=0.468)$. Hence, fixed-effect model was used to assess the association between uterine rupture and obstructed labor (Figure 5). 


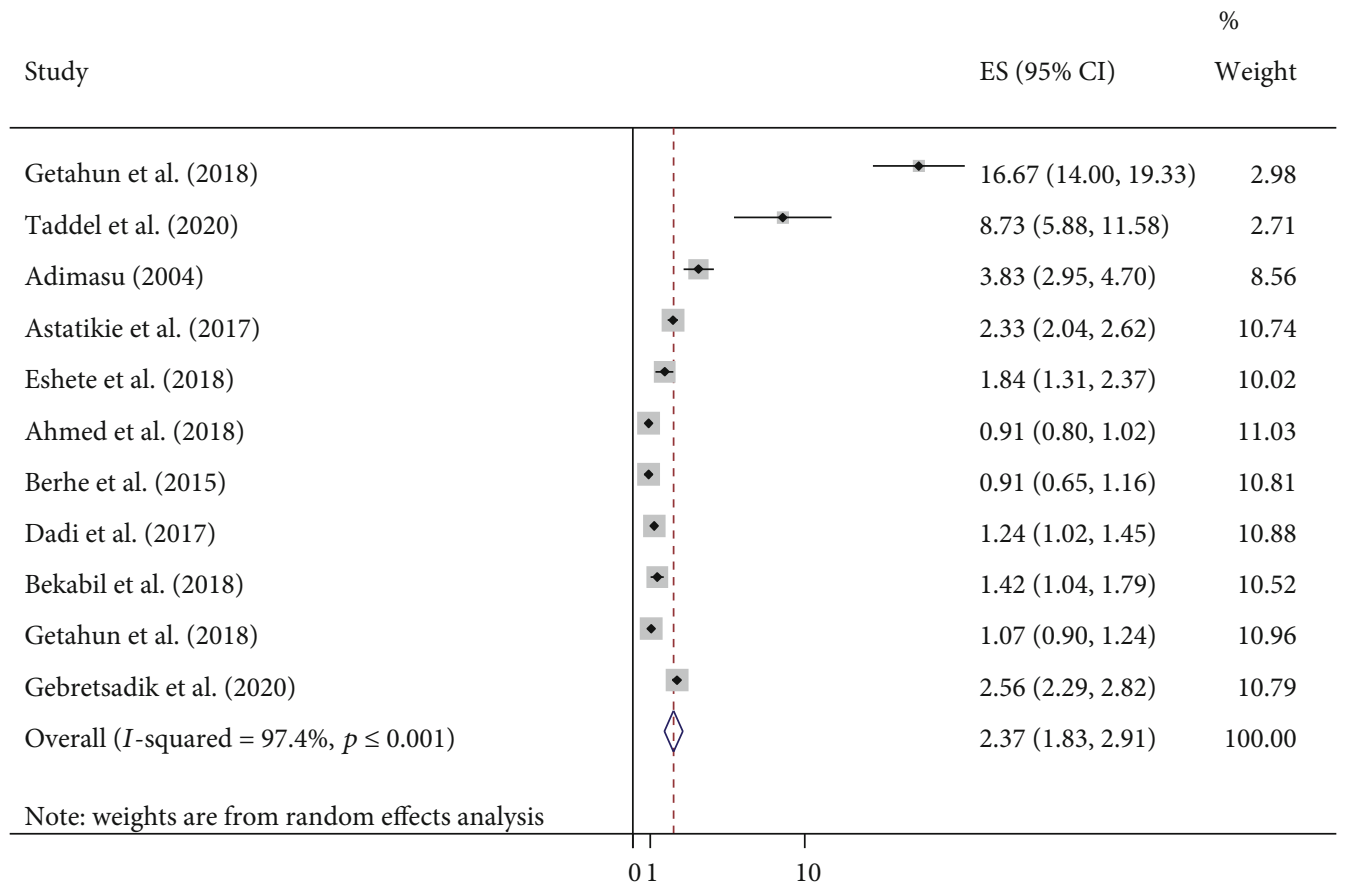

FIGURE 2: Forest plot showing the pooled prevalence of uterine rupture in Ethiopia.

TABLE 3: Shows subgroup analysis of the prevalence of uterine rupture by study characteristics in Ethiopia.

\begin{tabular}{lccc}
\hline Subgroup & $\begin{array}{c}\text { Number } \\
\text { of studies }\end{array}$ & Prevalence (95\% CI) & $I^{2}(\%)$ \\
\hline $\begin{array}{l}\text { Publication year } \\
\leq 2017\end{array}$ & 4 & $1.97(1.15,2.79)$ & 96.4 \\
After 2017 & 7 & $2.83(2.04,3.62)$ & 97.9 \\
Study design & & & \\
Cross-sectional & 8 & $2.97(2.22,3.71)$ & 98.2 \\
Case control & 2 & $1.28(1.09,1.47)$ & 0 \\
Cohort & 1 & $1.84(1.31,2.37)$ & \\
Region & & & \\
Amhara & 5 & $5.38(3.71,7.05)$ & 98.5 \\
Oromiya & 2 & $1.20(0.87,1.54)$ & 64.2 \\
SNNPR & 3 & $1.88(0.94,2.81)$ & 96.5 \\
Tigray & 1 & $0.91(0.65,1.16)$ & \\
Sample size & & & \\
$\leq 6000$ & 6 & $4.53(3.03,6.04)$ & 97.5 \\
$>6000$ & 5 & $1.61(1.03,2.19)$ & 97.9 \\
\hline
\end{tabular}

9.2. The Associations between Lack of ANC Follow-Up and Uterine Rupture. In this meta-analysis, six primary studies $[5,9,18,31,40,41]$ that examined the effect of lack of ANC follow-up on uterine rupture were included to assess the association between two variables. The finding of this meta-analysis indicated that lack of ANC follow-up was significantly associated with uterine rupture. Laboring mothers who did not have ANC follow-up during pregnancy was 5.79 times more likely developed uterine rupture as compared to those who had ANC follow-up $(\mathrm{OR}=5.79 ; 95 \% \mathrm{CI}=2.47$,
13.61). Marked heterogeneity was observed between the studies $\left(I^{2}=83.9 \%\right.$ and $\left.p \leq 0.001\right)$. Therefore, the association between uterine rupture and lack of ANC follow-up was determined by using random effects model (Figure 6).

9.3. The Association between Grand Multipara and Uterine Rupture. The association between grand multipara and uterine rupture was examined by using four primary studies that evaluated their association previously $[5,18,28,40]$. The finding of this meta-analysis showed that being grand multipara was significantly associated with the occurrence of uterine rupture in Ethiopia. Accordingly, the likelihood of uterine rupture occurrence was 10.79 times higher in grand multipara mothers as compared to those mothers who were para two to four $(\mathrm{OR}=10.79 ; 95 \% \mathrm{CI}=4.77,24.40)$. Moderate heterogeneity was seen between the included studies $\left(I^{2}=32.8 \%\right.$ and $\left.p=0.216\right)$. Hence, the associations between the two variables were examined by using random effects model (Figure 7).

9.4. The Associations between Duration of Labor Greater than 24 Hours and Uterine Rupture. In this systematic review, we assessed the effect of duration of labor greater than 24 hours and uterine rupture by using three studies [5, 28, 31]. The finding indicated that the duration of labor greater than 24 hours was significantly associated with uterine rupture in Ethiopia. Based on this finding, those mothers who had a duration of labor greater than 24 hours was 3.75 times more likely develop uterine rupture as compared to those who had a duration of labor less than 24 hours $(\mathrm{OR}=3.75$; $95 \% \mathrm{CI}=2.24,6.29)$. There was no heterogeneity across the studies $\left(I^{2}=0.0 \%\right.$ and $\left.p=0.790\right)$. Therefore, fixedeffect model was used to examine the association between these two variables (Figure 8). 


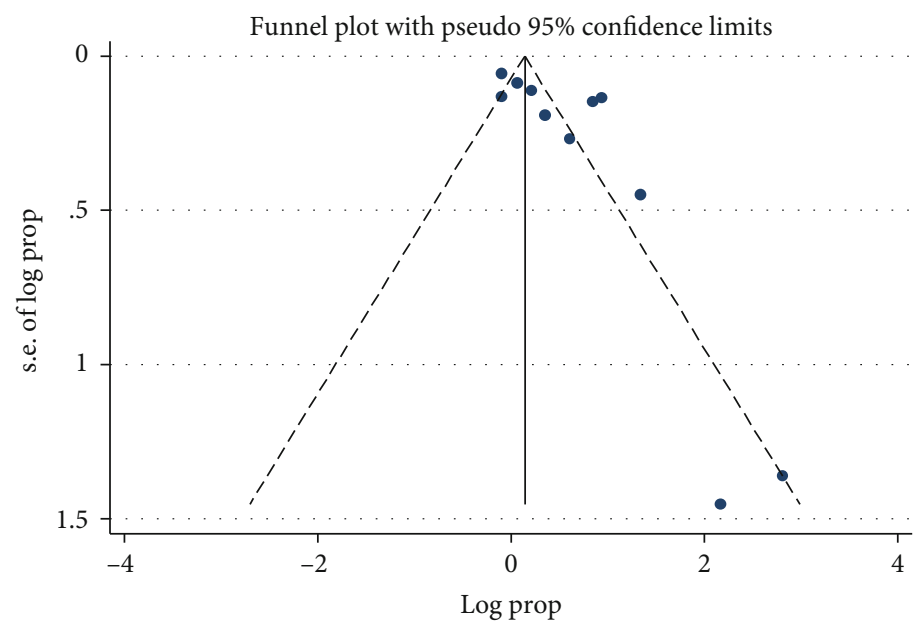

Figure 3: Funnel plots to assess publication bias of uterine rupture for 11 studies.

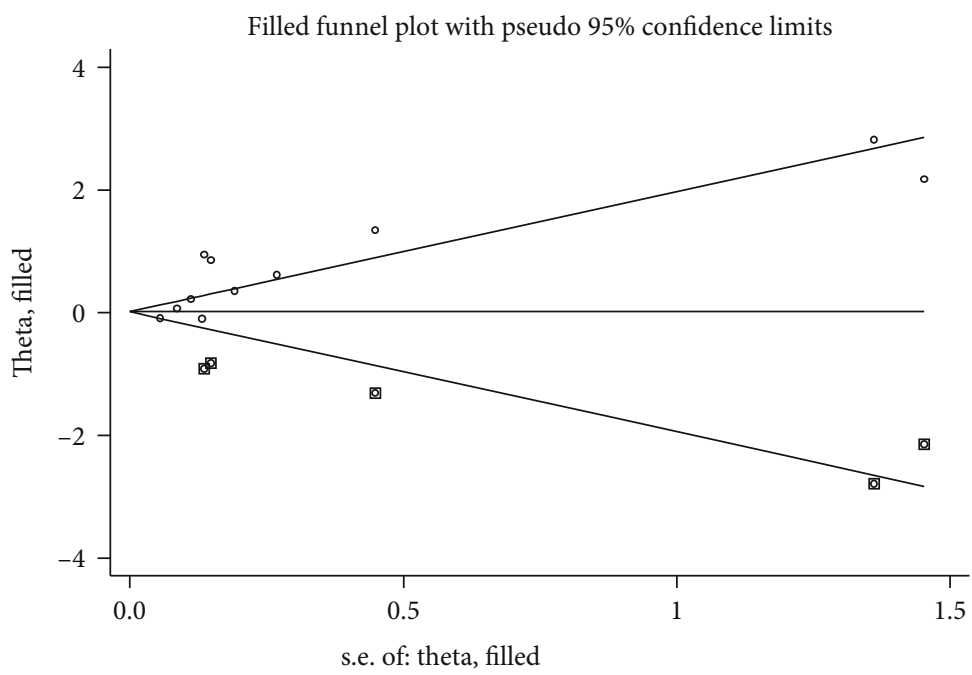

Figure 4: Funnel plots showing the results of trim and fill analysis for correcting publication. The circular dots demonstrate the observed studies, and the rectangular dots indicate the missing studies imputed by the trim and fill method.

TABLE 4: Adverse outcomes of uterine rupture in Ethiopia.

\begin{tabular}{lccccc}
\hline Adverse outcomes of uterine rupture & Model & Status of heterogeneity & Prevalence (95\% CI) & $I^{2}(\%)$ & $p$ value \\
\hline Anemia & Random & Marked heterogeneity & $64.34(33.06,95.61)$ & 99.5 & $\leq 0.001$ \\
Blood transfusion & Random & Marked heterogeneity & $52.30(11.24,93.36)$ & 98.5 & $\leq 0.001$ \\
Vesicovaginal fistula & Random & Marked heterogeneity & $7.03(3.19,10.88)$ & 86.6 & $\leq 0.001$ \\
Wound infection & Random & Marked heterogeneity & $17.67(5.90,29.43)$ & 97.1 & 0.093 \\
Maternal death & Random & Moderate heterogeneity & $3.44(1.87,5.01)$ & 46.9 & $\leq 0.001$ \\
Relaparotomy & Fixed & & $7.22(5.07,9.38)$ & 0 & 0.856 \\
Puerperal sepsis & Random & Moderate heterogeneity & $6.19(1.65,10.72)$ & 30.5 & $\leq 0.001$ \\
Stillbirth & Random & Moderate heterogeneity & $95.01(92.43,97.60)$ & 44.1 & 0.167 \\
\hline
\end{tabular}

9.5. The Association between Rural Residency and Uterine Rupture. The association between rural residency and uterine rupture was examined by using four primary studies $[9,18$, $31,41]$. The result showed that being a rural residency was significantly associated with the occurrence of uterine rup- ture in Ethiopia. Accordingly, the likelihood of uterine rupture occurrence was 7.17 times higher in those mothers who lived in the rural area as compared to those who lived in urban areas $(\mathrm{OR}=7.17 ; 95 \% \mathrm{CI}=3.90,13.16)$. There was moderate heterogeneity across those included studies 


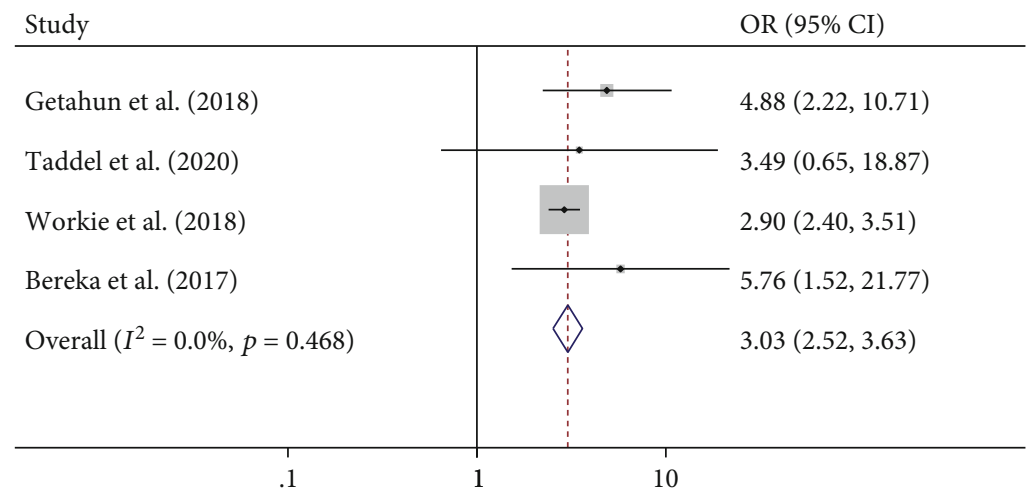

FiguRe 5: Forest plots showing the pooled odds ratio of the association between obstructed labor and uterine rupture.

\begin{tabular}{|c|c|c|}
\hline Study & & OR $(95 \% \mathrm{CI})$ \\
\hline Abebe et al. (2018) & & $9.64(4.37,21.28)$ \\
\hline Workie et al. (2018) & & $12.50(5.25,29.75)$ \\
\hline Eshete et al. (2018) & $\cdot$ & $1.30(0.72,2.36)$ \\
\hline Dadi et al. (2017) & & $3.17(1.65,6.09)$ \\
\hline Bekabil et al. (2018) & & $7.00(1.81,27.05)$ \\
\hline Bereka et al. (2017) & & $15.38(4.79,49.42)$ \\
\hline Overall $\left(I^{2}=83.9 \%, p \leq 0.001\right)$ & & $5.79(2.47,13.61)$ \\
\hline $\begin{array}{l}\text { Note: weights are from random } \\
\text { effects analysis }\end{array}$ & & \\
\hline
\end{tabular}

FIGURE 6: Forest plots showing the pooled effect of lack of ANC follow-up on uterine rupture in Ethiopia.

\begin{tabular}{|c|c|}
\hline Study & OR $(95 \% \mathrm{CI})$ \\
\hline Getahun et al. (2018) & $12.55(3.64,43.23)$ \\
\hline Abebe et al. (2018) & $27.89(8.42,92.36)$ \\
\hline Bekabil et al. (2018) & $4.37(1.05,18.21)$ \\
\hline Bereka et al. (2017) & $6.32(1.35,29.57)$ \\
\hline Overall $\left(I^{2}=32.8 \%, p=0.216\right)$ & $10.79(4.77,24.40)$ \\
\hline $\begin{array}{l}\text { Note: weights are from random } \\
\text { effects analysis }\end{array}$ & \\
\hline
\end{tabular}

FIGURE 7: Forest plots showing the pooled odds ratio of the association between uterine rupture and grand multipara.

$\left(I^{2}=54.7 \%\right.$ and $\left.p=0.085\right)$. As a result, random effects model was applied to evaluate the association between uterine rupture and rural residency (Figure 9).

\section{Discussion}

This systematic review and meta-analysis is aimed at estimating the pooled prevalence, determinants, and adverse outcomes of uterine rupture in Ethiopia. To the best of our knowledge, this meta-analysis is the first of its kind to esti- mate the pooled prevalence, determinants, and adverse outcomes of uterine rupture in Ethiopia. Estimating the pooled prevalence, determinants, and adverse outcomes of uterine rupture may enable policymakers and clinicians at different levels to design evidence-based prevention and intervention strategies.

This meta-analysis found that the pooled prevalence of uterine rupture in Ethiopia was 2.37\% (1.83 and 2.91). This finding was higher than a nationwide population-based study done in Belgium (0.036\%) [4], Sweden (0.091\%) [11], United 


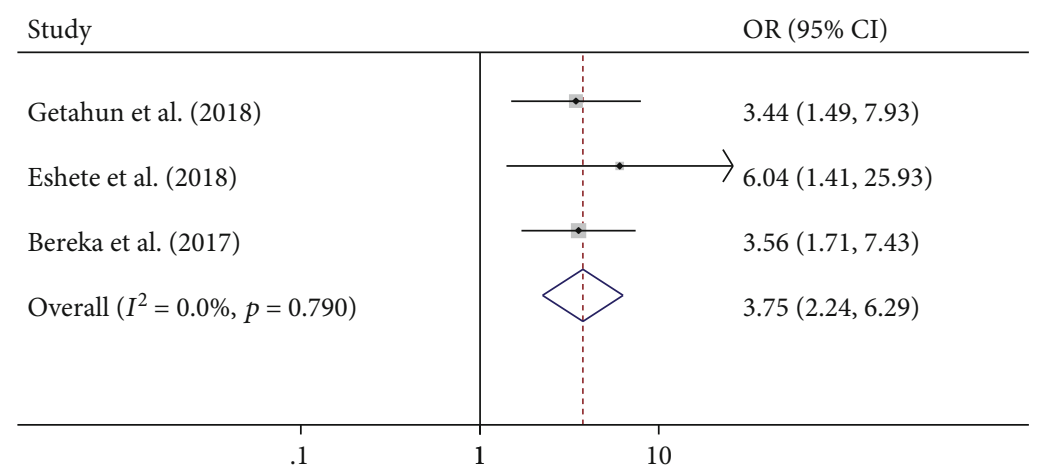

FIGURE 8: Forest plots showing polled odds ratio of the association between duration of labor $>24$ hours and uterine rupture.

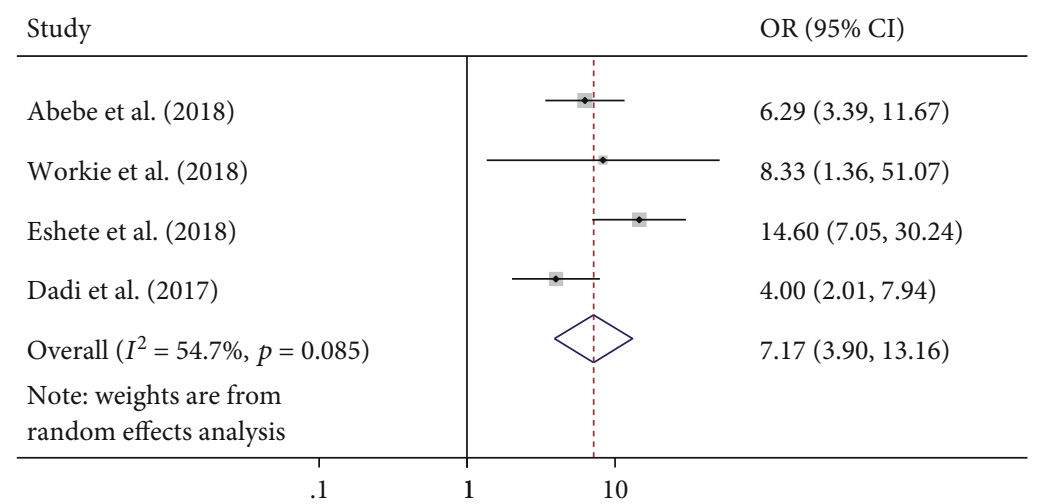

FIGURE 9: Forest plots showing the pooled odds ratio of the association between uterine rupture and rural residency.

Kingdom (0.019\%) [10], Netherlands (0.059\%) [12], and Norway (6.1 per 10,000 or $0.061 \%$ ) [42]. The variation could be due to inaccessibility of district and referral hospitals, difficult transportation systems, and difference in management and emergency referral of cases in our country. Beside to this, socioeconomic variation between the two countries, delay in deciding for referral and delay in giving immediate care for high-risk pregnant/laboring mothers in the study setting might also contribute to this variation. Furthermore, delay in seeking health services in Ethiopia due to sociocultural and religious issues, home delivery habit, family influence, lack of awareness on danger sign of labor and childbirth, inadequate birth preparedness plan, and poor quality of health services may also contributed to this difference [43].

This finding was also higher than the result of a large sampled study done in Senegal and Mali (0.67\%) [44]. This might be related to increasing tolerance to longer duration of labor in our country. Differences in maternal health service accessibility and resources disparities to manage obstetric emergency between the study settings might also contribute to this discrepancy. In addition, study design and sample size variation between the studies might also contribute to this difference. Moreover, the presences of sociocultural and religious issues, use of traditional options at homes, and inadequate birth preparedness plan in our setup might also contribute to this deviation [45].

The finding of subgroup analysis based on region indicated that the prevalence of uterine rupture was significantly variable across the regions. Accordingly, the highest preva- lence of uterine rupture was observed in Amhara with a prevalence of 5.38\% followed by Southern Nations, Nationalities, and Peoples Region (SNNPR) with a prevalence of $1.88 \%$, Oromiya region with $1.20 \%$, and then Tigray region with a prevalence of $0.91 \%$. The variation might be related to increasing number of studies addressing uterine rupture in the Amhara region (seven studies) as compared to the Tigray region (only two studies). As the number of studies increased, the prevalence might be increased in the region as a result of an increased sample size. Moreover, EDHS 2016 and mini EDHS 2019 indicated that maternal health service coverage such as ANC follow-up and institutional delivery was relatively better in Tigray region. As a result, the prevalence of uterine rupture may decrease in this region $[46,47]$. Beside to this, difference in health seeking behaviour between the regions as a result of variation in traditional belief, educational status, and home delivery habits may contribute to this variation. Moreover, there is better accessibility of infrastructures and transportation system (roads) in Tigray region; as a result, the prevalence of uterine rupture is low in Tigray region as compared to other regions.

In the present study, obstructed labor was significantly associated with uterine rupture. The likelihood of uterine rupture was 3.03 times higher in those laboring mothers who had obstructed labor as compared to those who did not have obstructed labor. This funding was supported by a study funding in Uganda [13], Senegal, and Mali [44]. This could be due to the presence of uncorrected mechanical obstruction that affects engagement and descent. In this 
condition, the uterus contract and retract for long periods and develop Bandl's ring and uterine rupture if action is not taken timely.

Lack of ANC follow-up was also another determinant factor of uterine rupture. Mothers who did not have ANC follow-up during pregnancy were 5.79 times more likely to develop uterine rupture as compared to those mothers who had ANC follow-up. This funding was in agreement with a study funding in Uganda [13, 48], Senegal, and Mali [44]. If the woman did not have ANC follow-up, predisposing factors for uterine rupture and any abnormalities or risks may not be identified early. Beside to this, a pregnant woman may not get adequate information or advice on danger signs of pregnancy and birth preparedness and complication redness plan including the sign of labor and timely arrival to health institutions when labor starts. This condition may facilitate the occurrence of uterine rupture.

According to this study, being grand multipara was significantly associated with uterine rupture. The occurrence uterine rupture was 10.79 times higher in grand multipara mothers as compared to those mothers who were para II-IV. This evidence was supported by a study funding in Uganda [49]. The possible justification for this association could be due to the weakening of the uterus in grand multiparous women [44]. Besides to this, it might be related to malpresentation, which is more likely occur in grand multiparous due to the lax uterus and weak abdominal wall [49].

Furthermore, duration of labor $>24$ hours was significantly associated with uterine rupture. Those laboring mothers who had a duration of labor $>24$ hours were 3.75 times more likely to develop uterine rupture as compared to those who had a duration of labor $\leq 24$ hours. This could be due to different obstetric interventions in case of prolonged labor. To shorten the duration of labor, different obstetric interventions might be applied (such as the use of oxytocin and operative delivery). This condition might lead to uterine rupture. Beside to this, the association between uterine rupture and prolonged labor may also be due to obstructed labor.

Finally, rural residency was significantly associated with the occurrence of uterine rupture. Accordingly, the likelihood of uterine rupture occurrence was 7.17 times higher in those mothers who lived in the rural areas as compared to those who lived in urban areas. This finding was supported by a study finding in Yemen [50], Senegal, and Mali [44]. The possible justification for this association might be due to delay in reaching health institutions because of the inaccessibility of nearby health facilities or distant health institutions and low levels of educational status in rural residency as compared to the urban areas. Moreover, there may be poor access to basic and compressive emergency obstetric care in the rural areas.

\section{Limitation of the Study}

This systematic review and meta-analysis has several limitations. All regions in Ethiopia were not represented in this systematic review and meta-analysis due to limited number of studies in the country (only four regions were represented in this study). As a result, the finding of this systematic review and meta-analysis may not exactly indicate the national prevalence of uterine rupture. Beside to this, almost all of the studies did not assess the relationship between previous caesarean scar and uterine rupture. Furthermore, the majority of the studies included in this systematic review and meta-analysis were cross-sectional in nature; as a result, the outcome variable might be affected by other confounding variables. Heterogeneity of the data obtained from the included studies was also another limitation for this study.

\section{Conclusion}

The overall prevalence of uterine rupture was high in Ethiopia. Obstructed labor, lack of ANC follow-up, duration of labor $>24$ hours, being grand multipara, and rural residency were determinants of uterine rupture. This study implies the need to develop plans and policies to improve antenatal care follow-up and labor and delivery management at each level of health system. Emphasis should be given to those women at a rural residency and grand multipara mothers (para $\geq 5$ ).

\section{Abbreviations \\ ANC: Antenatal care \\ EDHS: Ethiopian Demographic Health Survey \\ OR: Odds ratio \\ SNNPR: Southern Nations, Nationalities, and Peoples Region.}

\section{Data Availability}

All the datasets used in this study are accessible in the published article and its Additional files.

\section{Conflicts of Interest}

The authors state that they have no conflicts of interests.

\section{Authors' Contributions}

DA designed the study, participated in data analysis, and drafted the paper. MM, AM, and WN established the search strategy, performed the critical appraisal, participated in data analysis, and revised the manuscript. All authors read and approved the final manuscript before submitting it for publication.

\section{References}

[1] D. M. Ahmed, T. S. Mengistu, and A. G. Endalamaw, "Incidence and factors associated with outcomes of uterine rupture among women delivered at Felegehiwot referral hospital, Bahir Dar, Ethiopia: cross sectional study," BMC Pregnancy and Childbirth, vol. 18, no. 1, p. 447, 2018.

[2] S. O. Handady, H. H. Sakin, and A. A. M. Alawad, "Uterine rupture: a review of 15 cases at bandier maternity hospital in Somalia," Clinical Obstetrics, Gynecology and Reproductive Medicine, vol. 1, no. 2, pp. 55-58, 2015. 
[3] A. Gupta and S. Nanda, "Uterine rupture in pregnancy: a fiveyear study," Archives of Gynecology and Obstetrics, vol. 283, no. 3, pp. 437-441, 2011.

[4] G. Vandenberghe, M. De Blaere, V. Van Leeuw et al., "Nationwide population-based cohort study of uterine rupture in Belgium: results from the Belgian Obstetric Surveillance System," BMJ Open, vol. 6, no. 5, p. e010415, 2016.

[5] T. M. Bereka, A. M. Aweke, and T. E. Wondie, "Associated factors and outcome of uterine rupture at Suhul General Hospital, Shire Town, North West Tigray, Ethiopia 2016: a case-control study," Obstetrics and gynecology international, vol. 2017, Article ID 8272786, 7 pages, 2017.

[6] K. Motomura, T. Ganchimeg, C. Nagata et al., "Incidence and outcomes of uterine rupture among women with prior caesarean section: WHO Multicountry Survey on Maternal and Newborn Health," Scientific Reports, vol. 7, no. 1, 2017.

[7] N. Aziz and S. Yousfani, "Analysis of uterine rupture at university teaching hospital Pakistan," Pakistan journal of medical sciences., vol. 31, no. 4, pp. 920-924, 2015.

[8] H. L. Kidanto, I. Mwampagatwa, and J. Van Roosemalen, "Uterine rupture: a retrospective analysis of causes, complications and management outcomes at Muhimbili National Hospital in Dar es Salaam, Tanzania," Tanzania journal of health research, vol. 14, no. 3, 2012.

[9] T. L. Dadi and T. E. Yarinbab, "Estimates of uterine rupture bad outcomes using propensity score and determinants of uterine rupture in Mizan-Tepi University Teaching Hospital: case control study," Journal of Pregnancy, vol. 2017, Article ID 6517015, 9 pages, 2017.

[10] K. E. Fitzpatrick, J. J. Kurinczuk, Z. Alfirevic, P. Spark, P. Brocklehurst, and M. Knight, "Uterine rupture by intended mode of delivery in the UK: a national case-control study," PLoS medicine, vol. 9, no. 3, article e1001184, 2012.

[11] M. Kaczmarczyk, P. Sparén, P. Terry, and S. Cnattingius, "Risk factors for uterine rupture and neonatal consequences of uterine rupture: a population-based study of successive pregnancies in Sweden," BJOG: An International Journal of Obstetrics \& Gynaecology., vol. 114, no. 10, pp. 1208-1214, 2007.

[12] J. Zwart, J. Richters, F. Öry, J. De Vries, K. Bloemenkamp, and J. Van Roosmalen, "Uterine rupture in the Netherlands: a nationwide population-based cohort study," BJOG: An International Journal of Obstetrics \& Gynaecology., vol. 116, no. 8, pp. 1069-1080, 2009.

[13] I. Kadowa, "Ruptured uterus in rural Uganda: prevalence, predisposing factors and outcomes," Singapore Medical Journal, vol. 51, no. 1, pp. 35-38, 2010.

[14] V. A. Loue, J. M. Dia, D. N. Effoh et al., "Management and prognosis of uterine rupture during labor in an undermedicalized country: about 513 cases collected at the Cocody University Hospital Center (Abidjan-Cote d'Ivoire)," International Journal of Reproduction, Contraception, Obstetrics and Gynecology, vol. 10, pp. 1277-1282, 2015.

[15] G. J. Hofmeyr, L. Say, and A. M. Gülmezoglu, "SYSTEMATIC REVIEW: WHO systematic review of maternal mortality and morbidity: the prevalence of uterine rupture," BJOG: An International Journal of Obstetrics \& Gynaecology, vol. 112, no. 9, pp. 1221-1228, 2005.

[16] Organization WH, Trends in Maternal Mortality 2000 to 2017: Estimates by WHO, UNICEF, UNFPA, World Bank Group and the United Nations Population Division, 2019.
[17] A. Gebretsadik, H. Hagos, and K. Tefera, "Outcome of uterine rupture and associated factors in Yirgalem general and teaching hospital, southern Ethiopia: a cross-sectional study," BMC pregnancy and childbirth, vol. 20, no. 1, p. 256, 2020.

[18] F. Abebe, E. Mannekulih, A. Megerso, A. Idris, and T. Legese, "Determinants of uterine rupture among cases of Adama city public and private hospitals, Oromia, Ethiopia: a case control study," Reproductive health, vol. 15, no. 1, p. 161, 2018.

[19] P. Osemwenkha and J. Osaikhuwuomwan, "A 10-year review of uterine rupture and its outcome in the University of Benin Teaching Hospital, Benin City," Nigerian Journal of Surgical Sciences, vol. 26, no. 1, pp. 1-4, 2016.

[20] Y. Berhan and A. Berhan, "Causes of maternal mortality in Ethiopia: a significant decline in abortion related death," Ethiopian Journal of Health Sciences, vol. 24, p. 15, 2014.

[21] B. Ayele, H. Gebretnsae, T. Hadgu et al., "Maternal and perinatal death surveillance and response in Ethiopia: achievements, challenges and prospects," PloS one, vol. 14, no. 10, article e0223540, 2019.

[22] N. Rizwan, R. M. Abbasi, and S. F. Uddin, "Uterine rupture, frequency of cases and fetomaternal outcome," JPMA-Journal of the Pakistan Medical Association, vol. 61, no. 4, pp. 322-324, 2011.

[23] E. Assefa and A. Wesson, "Maternal outcome following uterine rupture and its associated factors among mothers who delivered at Felege-hiwot referral hospital, Amhara-Ethiopia," Women's Health, vol. 8, no. 2, pp. 143-148, 2019.

[24] T. O. Egbe, G. E. Halle-Ekane, C. N. Tchente, J. E. Nyemb, and E. Belley-Priso, "Management of uterine rupture: a case report and review of the literature," BMC research notes, vol. 9, no. 1, p. 492, 2016.

[25] M. A. Ahmed, G. E. S. Elkhatim, M. A. A. E. Ounsa, and E. Y. Mohamed, "Rupture uterus in Sudanese women: management and maternal complications," WORLD JOURNAL OF PHARMACY AND PHARMACEUTICAL SCIENCES, vol. 4, no. 4, pp. 1669-1675, 2015.

[26] G. A. Tessema, C. O. Laurence, Y. A. Melaku et al., "Trends and causes of maternal mortality in Ethiopia during 19902013: findings from the Global Burden of Diseases study 2013," BMC Public Health, vol. 17, no. 1, p. 160, 2017.

[27] B. Meshesha, G. Dejene, and T. Hailemariam, "The role of maternity waiting area in improving obstetric outcomes: a comparative cross-sectional study, Jinka zonal hospital, southern regional state," Journal of Women's Health Care, vol. 6, no. 6, 2017.

[28] W. T. Getahun, A. A. Solomon, F. Y. Kassie, H. K. Kasaye, and H. T. Denekew, "Uterine rupture among mothers admitted for obstetrics care and associated factors in referral hospitals of Amhara regional state, institution-based cross-sectional study, Northern Ethiopia, 2013-2017," PLoS One, vol. 13, no. 12, article e0208470, 2018.

[29] G. Astatikie, M. A. Limenih, and M. Kebede, "Maternal and fetal outcomes of uterine rupture and factors associated with maternal death secondary to uterine rupture," BMC pregnancy and childbirth, vol. 17, no. 1, p. 117, 2017.

[30] Y. Berhe, H. Gidey, and L. L. Wall, "Uterine rupture in Mekelle, northern Ethiopia, between 2009 and 2013," International Journal of Gynecology \& Obstetrics, vol. 130, no. 2, pp. 153-156, 2015.

[31] A. Eshete, S. Mekonnen, and F. Getachew, "Prevalence and factors associated with rupture of gravid uterus and FETO- 
maternal outcome: a one-year retrospective cohort study," Ethiopian Medical Journal, vol. 56, p. 1, 2018.

[32] M. Taddele, A. Talie, and M. Admas, "Assessment of uterine rupture and its associated factors among women managed for obstetric care in Debre Markos Referral Hospital, Amhara Region, Northeast, Ethiopia, 2018," Acta Scientific Women's Health, vol. 2, no. 3, 2020.

[33] A. ADMASSU, "Analysis of ruptured uterus in Debre Markos hospital, Ethiopia,” East African Medical Journal, vol. 81, no. 1, 2004.

[34] T. Getahun, D. Workneh, C. Hailu, and B. T. Gashaw, "The prevalence and outcomes of uterine rupture in Wolliso St. Luke Catholic Hospital, Oromia Regional State, Southwest Shewa, Ethiopia," Medical Journal of Obstetrics and Gynecology, vol. 6, no. 1, 2018.

[35] J. Peterson, V. Welch, M. Losos, and P. Tugwell, The Newcastle-Ottawa Scale (NOS) for Assessing the Quality of Nonrandomised Studies in Meta-Analyses, Ottawa Hospital Research Institute, Ottawa, 2011.

[36] J. P. Higgins, S. G. Thompson, J. J. Deeks, and D. G. Altman, "Measuring inconsistency in meta-analyses," BMJ (Clinical research ed), vol. 327, no. 7414, pp. 557-560, 2003.

[37] M. Borenstein, L. V. Hedges, J. P. T. Higgins, and H. R. Rothstein, "A basic introduction to fixed-effect and random-effects models for meta-analysis," Research synthesis methods, vol. 1, no. 2, pp. 97-111, 2010.

[38] R. DerSimonian and R. Kacker, "Random-effects model for meta-analysis of clinical trials: an update," Contemporary Clinical Trials, vol. 28, no. 2, pp. 105-114, 2007.

[39] J. L. Peters, A. J. Sutton, D. R. Jones, K. R. Abrams, and L. Rushton, "Comparison of two methods to detect publication bias in meta-analysis," Journal of the American Medical Association, vol. 295, no. 6, pp. 676-680, 2006.

[40] T. T. Bekabil, "Uterine rupture among women who gave birth at Nekemte Referral Hospital: case control study," Biology and Medicine, vol. 10, no. 2, 2018.

[41] A. Workie, Y. Getachew, K. Temesgen, and P. Kumar, “Determinants of uterine rupture in Dessie Referral Hospital, North East Ethiopia, 2016: case control design," International Journal of Reproduction, Contraception, Obstetrics and Gynecology, vol. 7, no. 5, pp. 1712-1717, 2018.

[42] I. Al-Zirqi, B. Stray-Pedersen, L. Forsén, A. K. Daltveit, and S. Vangen, "Uterine rupture: trends over 40 years," BJOG: An International Journal of Obstetrics \& Gynaecology., vol. 123, no. 5, pp. 780-787, 2016.

[43] Y. Berhan and A. Berhan, "Commentary: reasons for persistently high maternal and perinatal mortalities in Ethiopia: part III-perspective of the "three delays" model," Ethiopian Journal of Health Sciences., vol. 24, pp. 137-148, 2014.

[44] R. Delafield, C. M. Pirkle, and A. Dumont, "Predictors of uterine rupture in a large sample of women in Senegal and Mali: cross-sectional analysis of QUARITE trial data," BMC Pregnancy and Childbirth, vol. 18, no. 1, p. 432, 2018.

[45] A. G. Worku, A. W. Yalew, and M. F. Afework, "Maternal complications and women's behavior in seeking care from skilled providers in North Gondar, Ethiopia," PLOS ONE, vol. 8, no. 3, p. e60171, 2013.

[46] Agency Cs, Ethiopian mini Demographic and health Survey, 2019, https://dhsprogram.com/pubs/pdf/PR120/PR120.pdf.

[47] Agency cs, ETHIOPIA Demographic and Health Survey, Central Statistical Agency and ICF, Addis Abeba, 2016.
[48] J. Wandabwa, P. Doyle, J. Todd, P. Kiondo, M. A. Wandabwa, and F. Azinga, "Risk factors for ruptured uterus in Mulago Hospital Kampala, Uganda," East African Medical Journal, vol. 85, no. 2, pp. 56-63, 2008.

[49] P. K. Mukasa, J. Kabakyenga, J. K. Senkungu, J. Ngonzi, M. Kyalimpa, and V. J. Roosmalen, "Uterine rupture in a teaching hospital in Mbarara, western Uganda, unmatched case- control study," Reproductive health, vol. 10, no. 1, 2013.

[50] H. A. Al Shamahy, "Uterine rupture and associated factors during labor amongst women delivered in Saudi Hajjah Hospital in Hajjah City North West Yemen," World Journal of Gynecology \& Womens Health, vol. 1, no. 2, 2018. 